Reprod. Nutr. Dévelop., 1985, 25 (1 B), 341-344.

\title{
Mobilisation des réserves corporelles chez la truie primipare en lactation
}

\author{
M. ETIENNE, J. NOBLET, B. DESMOULIN
}

Station de Recherches sur l'Elevage des Porcs, I.N.R.A., St-Gilles, 35590 L'Hermitage, France.

Summary. Mobilization of body stores in the lactating primiparous sow.

To determine the nature and the amount of the tissues involved in weight loss during lactation, an experiment was carried out on 9 series of 3 littermate Large-White gilts divided into three groups. The animals were similarly raised until first farrowing. The control gilts were slaughtered at farrowing, while those in the other two groups (group $\mathrm{H}$ and group B) received diets differing only in energetic level (14.2 and 10.3 Mcal ME/day, respectively). The gilts were slaughtered after 22 days of lactation. Half-carcasses were cut and dissected. Muscle weight decreased similarly in the two lactating groups $1-9.5$ and - $11.3 \mathrm{~kg}$ in groups $\mathrm{H}$ and $\mathrm{B}$, respectively), but fat loss was higher in the energy-restricted animals $(-6.7 \mathrm{vs}-1.7 \mathrm{~kg})$. These results were confirmed by changes in adipose cell size, blood urea and plasma free fatty acids between farrowing and weaning.

\section{Introduction.}

Au cours de son cycle de reproduction, la truie subit généralement des variations de poids importantes : à un gain pondéral de gestation succède une perte de poids pendant la lactation. Comme dans d'autres espèces domestiques (Chilliard et al., 1983), cette observation a abouti au concept de " réserves corporelles " édifiées avant la parturition et utilisées ultérieurement pour la synthèse du lait. Cependant, la nature, la localisation et l'importance quantitative des tissus mis en cause dans ces phénomènes de mobilisation sont encore mal connues chez la truie (Etienne et al., 1982 ; Duée et al., 1983). Aussi la présente étude a-telle été entreprise dans le but de préciser les connaissances dans ce domaine.

\section{Matériel et méthodes.}

L'expérience porte sur neuf blocs de trois truies nullipares Large-White, alimentées de façon identique pendant leur croissance et leur première gestation. Pen- 
dant une lactation de 22 jours, deux d'entre elles sont maintenues avec leurs portées égalisées à 10 porcelets en chambres respiratoires de façon à étudier leur métabolisme énergétique. Ces animaux reçoivent des régimes ne différant que par les apports énergétiques (14,2 Mcal EM/jour : lot H ; 10,3 Mcal EM/jour : lot B), tous les autres nutriments (protéines, acides aminés, minéraux, vitamines) étant fournis de façon identique dans les deux lots. Pendant la gestation et la lactation, on suit l'évolution de la taille des adipocytes du tissu gras sous-cutané, de l'activité lipolytique mesurée in vitro sur adipocytes isolés à la collagénase, de l'urémie et du taux d'acides gras non estérifiés plasmatiques. Les truies allaitantes sont abattues le jour du sevrage tandis que la troisième femelle de chaque bloc servant de témoin initial, est sacrifiée à la parturition. La demi-carcasse droite de ces animaux est découpée puis disséquée en quatre fractions: peau, graisses externes + graisses internes, muscles (comportant également les graisses intermusculaires), os.

\section{Résultats et discussion.}

La restriction alimentaire des truies n'affecte pas leur production d'énergie dans le lait, qui est de $8,2 \mathrm{Mcal} /$ jour en moyenne. Toutes les femelles se trouvent en bilan énergétique négatif, et ce d'autant plus qu'elles sont davantage rationnées $(-2,12$ et $-5,42 \mathrm{Mcal} /$ jour respectivement dans les lots $H$ et $B)$. II en résulte une perte pondérale pour tous les animaux, surtout dans le lot $B(-673$ et $-1136 \mathrm{~g} /$ jour dans les lots $H$ et $B$ ).

L'estimation des variations de poids des fractions disséquées chez les femelles allaitantes à partir de la composition corporelle des animaux abattus à la parturition montre que leur masse musculaire diminue d'environ $13 \%$ indépendamment du niveau de restriction énergétique (tabl. 1). Au contraire, le poids des graisses disséquées est affecté de façon significativement plus importante chez les animaux les plus carencés en énergie $(-25 \%$ dans le lot $B$ ). Ces résultats sont corroborés par les variations des paramètres biochimiques et morphologiques étudiés : l'urémie s'accroît de façon comparable dans les deux lots au cours de la lactation, traduisant une mobilisation protéique similaire chez toutes les truies. Le volume moyen des adipocytes du tissu gras sous-cutané dorsal ne diminue de façon significative entre la parturition et le sevrage que chez les truies du lot $B$,

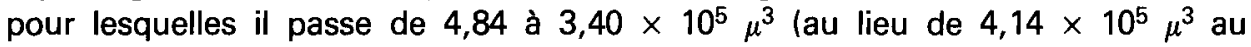
sevrage chez les femelles du lot $\mathrm{H}$ ). Enfin, le taux d'acides gras non estérifiés plasmatiques est significativement plus élevé à 0 et 9 jours de lactation dans le lot $\mathrm{B}$ que dans le lot $\mathrm{H}$; il augmente au sevrage chez ces dernières. La mobilisation des graisses corporelles intervient donc beaucoup plus précocément chez les animaux les plus rationnés. A la différence des résultats obtenus chez la vache allaitante (Jaster et Wegner, 1981), l'activité lipolytique in vitro des adipocytes du tissu gras sous-cutané dorsal n'est pas accrue apiès la parturition, qu'il s'agisse des activités basale ou stimulée par des doses croissantes d'adrénaline en présence de théophylline.

Chez la truie primipare en lactation, les réserves adipeuses ne sont donc partiellement mobilisées que dans le cas d'un déficit énergétique sévère, tandis que 
TABLEAU 1

Modifications de composition tissulaire des truies primipares pendant la lactation en fonction du niveau de rationnement énergétique.

\begin{tabular}{|c|c|c|c|c|}
\hline & \multirow{2}{*}{$\begin{array}{l}\text { Poids } \\
\text { à la parturition }\end{array}$} & \multicolumn{2}{|c|}{ Variations de poids } & \multirow{2}{*}{$\begin{array}{l}\text { Signification } \\
\text { stat. (1) }\end{array}$} \\
\hline & & Lot $\mathrm{H}$ & Lot B & \\
\hline \multicolumn{5}{|l|}{ Muscles + gras intermus- } \\
\hline Graisses $\ldots \ldots \ldots \ldots \ldots$ & 27,28 & $\begin{array}{l}-9,54 \\
-1,65\end{array}$ & $\begin{array}{r}-\quad 11,25 \\
-\quad 6,79\end{array}$ & * $(1,21)$ \\
\hline & 11,38 & $-0,72$ & $\begin{array}{l}-0,37 \\
-\end{array}$ & NS $(0,70)$ \\
\hline Peau ... & 6,49 & $-0,03$ & $-\quad 0,04$ & NS $(0,11)$ \\
\hline
\end{tabular}

(1) Différences entre les lots $H$ et $B$. NS : non significative ; ${ }^{*}: P<0,05$.

(1) Ecart-type moyen résiduel.

les muscles semblent mis à contribution de façon systématique. Ceci pourrait s'expliquer par une carence en protéines alimentaires, mais aussi par une certaine inadaptation des tissus adipeux à faire face à un déficit énergétique modéré. Ces résultats, en accord avec les observations de Duée et al. (1983) faites sur le même type d'animal placé dans des conditions nutritionnelles différentes, ne semblent cependant pas pouvoir être généralisés aux truies multipares dont les réserves adipeuses se trouvent extrêmement réduites après plusieurs cycles de reproduction (Whittemore et al., 1980 ; Etienne et al., 1982).

10 Réunion du groupe Développement I.N.R.A., Rennes, 9-10 mai 1984.

\section{References}

CHILLIARD Y., RÉMOND B., SAUVANT D., VERMOREL M., 1983. Particularités du métabolisme énergétique. In " Particularités nutritionnelles des vaches à haut potentiel de production ». Bull. tech., CRZV-Theix-INRA, 53, 37-64.

DUÉE P. H., DESMOULIN B., ETIENNE M., DURAND G., 1983. Effets d'une réduction de l'apport protéique pendant la gestation sur le métabolisme maternel et l'évolution de la composition corporelle au cours du cycle de reproduction chez la truie. Ann. Zootech., 32, 21-42.

ETIENNE M., PICHOT J. P., DESMOULIN B., 1982. Modifications de la composition corporelle des truies multipares au cours du cycle de reproduction. Effets du niveau d'alimentation en lactation. Journ. Rech. porcine en France, 14, 97-107.

JASTER E. H., WEGNER T. N., 1981. Beta-adrenergic receptors involvement in lipolysis of dairy cattle subcutaneous adipose tissue during dry and lactating state. J. Dairy Sci., 64, 16551663.

WHITTEMORE C. T., FRANKLIN M. F., PEARCE B. S., 1980. Fat changes in breeding sows. Anim. Prod., 31, 183-190. 